\title{
Performance evaluation of wireless networks with power saving support
}

\author{
Agashe A.A.* and Bodhe S.K. \\ ${ }^{*}$ Department of Electronics, JJMCOE, Jaysingpur, Maharashtra, India \\ Department of Electronics, BVCOE, Pune, Maharashtra, India
}

\begin{abstract}
Ad hoc networks, a subset of wireless networks, allow the formation of a wireless network without the need for access point. All participating users in an ad hoc network agree to accept and forward messages, to and from each other. Mobility management with reference to handoff management has widely been recognized as one of the most important and challenging issue in ad hoc network. Mobility of node is defined by various mobility models. Evaluating mobility models within an ad hoc network gives solution to find out performance measures like blocking probability, handoff probability, dropping probability, hop count, delay etc. This research is an investigation in search of the effect of Random Walk, Random Waypoint and Gauss Markov mobility models on performance of mobile ad hoc network. Power of nodes in ad hoc networks is supplied with batteries hence power-saving is an important issue. This paper also focuses on energy calculations.
\end{abstract}

Keywords- mobility models, handoff, dropping probability, blocking probability

\section{Introduction}

Mobile networks allow users to access services while on the move so giving end users freedom in terms of mobility. However, this freedom does bring uncertainties to mobile systems. Wireless networks are very popular due to their flexible nature, and the inherent possibility for wireless nodes to be mobile. Currently most wireless networks are infrastructured networks, where all communications go through an access point (AP) that acts as a gateway between the wired and wireless domains. To accommodate mobility, hand-over can be performed between two access points as the wireless station moves from the coverage area of one access point to another, enabling the communication to seamlessly continue. Infrastructure-less, or ad hoc networks have gained a lot of interest in the research community. In ad hoc networks there are no fixed routers or base stations, but instead all nodes have the capability to forward packets for each other. Handoff is the essential component for dealing with the mobility of end users. Handoff can be defined as opportunistic switching of mobile user's connections as they move and change their attachment points to the network. It guarantees the continuity of the wireless services when the mobile user moves across boundaries of their respective service areas. The ad hoc mobility models are the continuous time stochastic process, which characterizes the movement of nodes in twodimensional spaces. According to the movement pattern of each type, each node movement consists of sequence of random length interval, during which a node moves in constant speed and constant direction. The speed and direction of each node varies according to various mobility models. In the performance evaluation of handoff algorithm for wireless networks, the handoff algorithm should be tested under realistic conditions and realistic movements of the mobile user. The Synthetic mobility models attempt to realistically represent the behaviors of mobile nodes.

\section{Mobility models}

Mobile ad hoc networks are often studied through simulation and their performance can heavily depend on mobility model that govern the movement of node. A mobility model should attempt to mimic the movements of real mobile node (MN), changes in speed and direction must occur and they must occur in reasonable time slots. Random Walk Mobility Model, Random Waypoint Mobility Model, Random Direction Mobility Model, A Boundless Simulation Area Mobility Model, Gauss Markov Mobility Model are some of the entity mobility models which are used in the wireless network simulation [1].

\section{Random Walk Mobility Model}

Einstein first described the Random walk mobility model mathematically in 1926. Since many entities in nature move in extremely unpredictable ways, the Random Walk mobility model was developed to mimic this erratic movement.

\section{Random Waypoint Mobility Model}

The Random waypoint mobility model includes pause times between changes in direction and/or speed. A mobile node begins by staying in one location for a certain period of time. Once this time expires, the mobile node chooses a speed that is uniformly distributed between (Minspeed,Maxspeed). Mobile nodes then travels towards the newly chosen destination at the selected speed. Upon arrival, the mobile node 
pauses for a specified time period before starting the process again.

\section{Gauss-Markov Mobility Model}

The Gauss-Markov Mobility Model was designed to adapt to different levels of randomness via one tuning parameter. Initially each mobile node is assigned a current speed and direction. At fixed intervals of time, $n$, movement occurs by updating the speed and direction of each mobile node. Specifically, the value of speed and direction at the $n^{\text {th }}$ instant is calculated based upon the value of speed and direction at the $(n-$ $1^{\text {th }}$ instance and a random variable using the following equations:

$s_{n}=\alpha s_{n-1}+(1-\alpha) \bar{s}+\sqrt{\left(1-\alpha^{2}\right)} s_{x_{n-1}} \ldots \ldots \ldots \ldots$

$d_{n}=\alpha d_{n-1}+(1-\alpha) \bar{d}+\sqrt{\left(1-\alpha^{2}\right)} d_{x_{n-1}}$

Where $s_{n}$ and $d_{n}$ are the new speed and direction of the mobile node at time interval $n$; $\alpha$, where $0 \leq \alpha \leq 1$ is the tuning parameter used to vary the randomness, $s$ and $d$ are the constants representing the mean value of speed and direction as $n$ and $s_{x_{n-1}}$ and $d_{x_{n-1}}$ are random variables from Gaussian distribution. Totally random values (or Brownian motion) are obtained by varying the value of $\alpha=0$ and linear motion is obtained by setting $\alpha=1$. Intermediate levels of randomness are obtained by varying value of $\alpha$ between 0 and 1 .

\section{Hypothesis for simulation}

This includes design of Network Model, mobility model, traffic model, transmission model, preliminaries, framework of performance measures, etc.

\section{Network Model}

The wireless mobile ad hoc network (MANET) is modeled as a set of wireless nodes deployed in predetermined two dimensional rectangular area which is called as 'deployment region' or ' 0 ' order region. There are ' $m$ ' mobile nodes, which are distributed uniformly at, random over the 'deployment region' at $t=0$. Each node has unique ID and it is aware of its own position. For the purpose of mobility management the deployment region is partitioned into four squares. Fig 1 shows the partitioning scheme. As illustrated, the deployment region i.e. '0' order region is partitioned into $2 \times 2$ square regions; each of them is $1^{\text {st }}$ order region. $1^{\text {st }}$ order regions are further partitioned into $2 \times 2$-order regions; each of them is called as $2^{\text {nd }}$ order region. A second order region of each first order region, which lies in center of $0^{\text {th }}$ order region, is of our special interest. This region is called as handoff region. The nodes, which are present in handoff region, are called as relays. To identify the transmission range of each node, the hypothetical circles have to be drawn, by considering each node as the center of the circle. The transmission range of each node is identical. For the purpose of simulation we have considered that mobile nodes are moving in the deployment region with the mobility models which are described in section 2 .

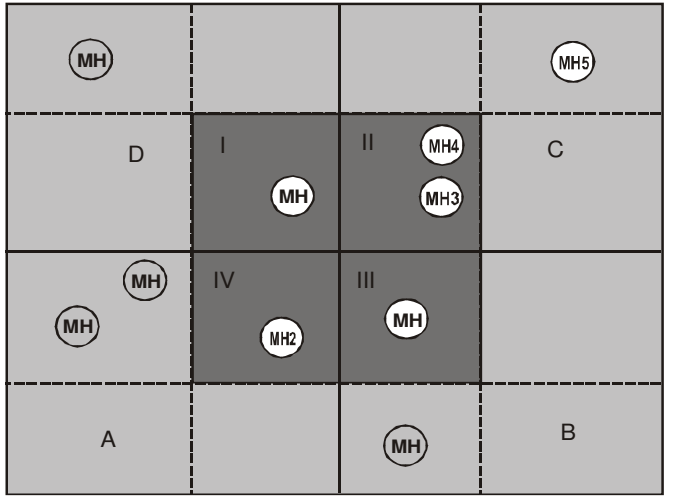

Fig 1-Simulation area showing partitioning scheme

\section{Traffic Model}

It is important to establish a traffic model before analyzing the system. Several traffic models have been established based on different assumptions about node mobility. The traffic model, which is used in simulation, is based on "Xie and Kuke's" [2] one and two dimensional traffic model. This model assumes uniformly distributed mobile nodes throughout an area and that a node is equally likely to move in any direction with respect to area border. From this assumption arrival rate of handoff is

$\lambda_{H}=E[c] \mu_{c}-d w e l l ;$

Here $E[c]$ is the average number of packets in the transmission range of the node and $\mu_{c}-d w e l l$ is the outgoing rate of packets from transmission range of node.

\section{Transmission Model}

Transmission Model used is Two Ray Ground Model that improves the principle of functioning of free space. The only real parameter is the node's distance $d$. TRG (Two Ray Ground Model) is a model that improves the principle of functioning of free space. The signal propagation model concerned keeps into account both the direct path between source and receiver and the ground reflection path. Hence, the additional feature of TRG is that ground reflection negatively affects the receiving power due to multi-path effect. For nodes within certain threshold distance the model behaves as free space. In contrast when nodes are far apart from each other for more than this threshold distance, 
then receiving signal strength (RSS) is inversely proportional to $d^{4}$.

$$
P_{r}(d)=P_{o} d^{-\alpha}
$$

For free space propagation model $\alpha=2$ and for TRG $\alpha=4$ where $\alpha$ is distance power gradient and $\operatorname{Pr}(d)$ is the received signal strength (RSS) of mobile node at distance' $d$ '. The user's location and RSS is monitored at every second.

\section{Preliminaries}

In our scenario following postulates are used to ensure bounded delay.

1. To provide good delay guarantees, it is necessary to assume that position of the destination is fixed. Indeed, if the destination is a mobile node, it will be impossible to provide any guarantee on delay. Hence it is assumed that source and destination are static nodes at the time of transmission, for packet duration.

2. The number of relays per packet should not be too large.

3. To ensure small delay, we must ensure that a packet does not lose its way along a path.

4. The mobile nodes know the direction in which they are moving up to some degree of accuracy.

5. A mobile node knows how far it will move in a line before changing the direction. This is true in random waypoint model, random walk model, and also in real situations where nodes know their destination.

6 . The mobile nodes relay the packet intended for destination among themselves such that packet moves closer and closer to the destination.

\section{Handoff Management Scheme}

This is the description and analysis of handoff algorithm. For every data packet carried by a mobile node, there is a point beyond which the packet is not handed to another node, it starts moving away from the destination.

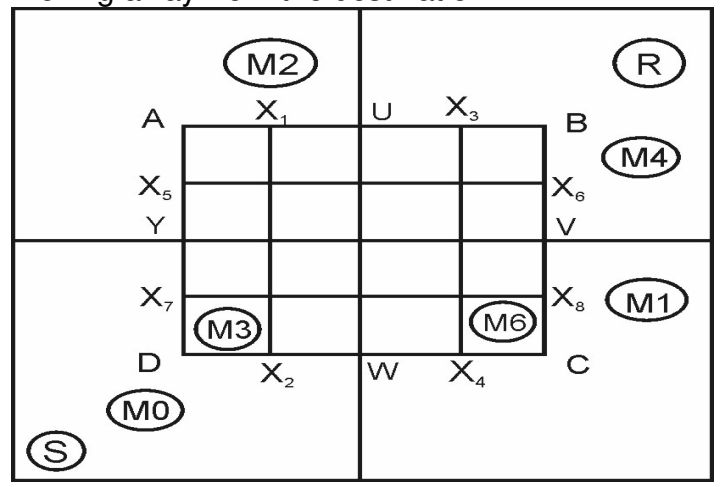

Fig 2- Situation of nodes to illustrate handoff algorithm
This leads to the notion of a handoff region. It is necessary to make sure that every packet is handed off to another mobile node within its handoff region. In the figure 2 source node $S$ wants to transmit data to destination $\mathrm{R}$. The mobile node $M_{0}$ has handed a packet from node S. Now $M_{0}$ carries the packet until it reaches in handoff region. If $M_{0}$ does not enter the handoff region and moves away from handoff region, it tries to handoff packet to another mobile node $M_{3}$ which is in the handoff region (i.e. rectangle ABCD) and moving towards $R$.

6. Scheduling: In order to handle packets for handoff, a deadline is defined in handoff region. The $X_{1}-X_{2}, X_{3}-X_{4}, X_{5}-X_{6}, X_{7}-X_{8}$ lines in figure 5 are termed as dead lines for node. The packet is said to be in handoff mode if, mobile node carrying that packet is in the handoff region and has not crossed above said deadlines. If mobile node $M_{0}$ is unable to handoff the packet to another mobile until it reaches the deadline, the packet is dropped. If mobile node $M_{0}$ handovers the packet to another mobile node until it reaches the deadline, but for further transmission of packet it doesn't get any relay then also the packet is dropped

\section{Handoff Algorithm using Received Signal Strength Measurements}

Handoff algorithm used here is based on measurement of received signal strength [3]. Let ' $A$ ' is the node which is carrying the packet to be transmitted. By knowing the current location of node ' $A$ ', the Received Signal Strength (RSS) of all adjacent nodes at node ' $A$ ' is measured. The node for which the packet has maximum RSS is the present node ' $A$ '. At the next position the RSS of all adjacent nodes of node ' $A$ ' is measured again and the one with maximum RSS is taken as next node in the packet transmission. The node ' $A$ ' measures the signal strength from the number of adjacent nodes. From all the measured data at each sample point the handover algorithm decides whether a handover should be made or not, if a handover should be made a new node should be selected. If node ' $A$ ' measures the signal strength from ' $m$ ' different nodes the hand off decision can be most general way be described as,

$b(n)=f\left(B_{0}(n), B_{1}(n), \ldots \ldots \ldots B_{m-1}(n)\right) \ldots \ldots \ldots(5)$

where

$b(n) \in(0,1, \ldots \ldots \ldots \ldots \ldots \ldots . . . M-1)$ 
Where $B_{i}(n)$ is the sequence of samples from node number $i$ up to sample number $n$. The function $f$ is evaluated at each sample and result $b(n)$ is the handover decision. If $b(n)=b(n-1)$ no handover is made, on the other hand if $b(n) \neq b(n-1)$ a handover is made to node $b(n)$. First the signal strength threshold $\beta$ is defined such that if signal strength is below $\beta$ it is not possible to send packet to chosen node. This is the situation when packet blocks. The packet blocking probability is described as

$$
P_{b}=P_{r}\left\{R S S_{b(n)}(n+1)<\beta\right\}
$$

A packet that is not blocked of course enters service but its ultimate fate has two possible outcomes. One is that its transmission is completed satisfactorily. The other is that the packet transmission is forced to terminate prematurely because it experiences unsuccessful handoff attempt prior to complete the transmission. This probability is called as packet dropping probability. The handoff is called as successful handoff if packet is delivered from mobile node $M_{i}$ to mobile node $M_{j}$ such that packet was in the handoff mode at the time of delivery and it was delivered before deadline. The probability of such successful handoff is called as handoff probability

$$
P_{H}=P_{r}\{b(n) \neq b(n-1)\} \ldots \ldots \ldots(7)
$$

\section{Performance Measures}

Handoff probability $\left(P_{H}\right)$ : The handoff is called as successful handoff if packet is delivered such that it was in the handoff mode at the time of delivery and delivered before deadline. The probability of such successful handoff is called as handoff probability.

\section{Results of Simulation Model}

We have incorporated the mobility models in simulation model. The simulation is carried out to find the handoff probability.

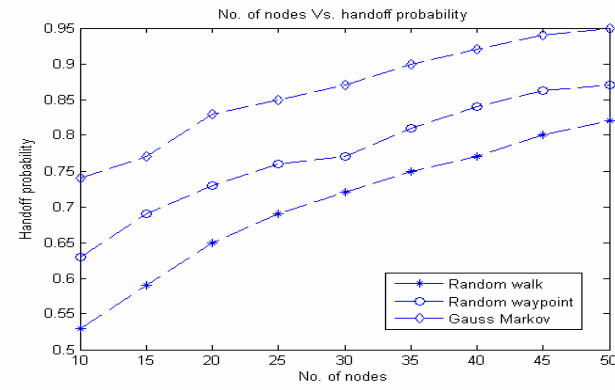

Fig 3- Handoff probability verses number of nodes
Figure 3 shows handoff probability with respect to number of nodes incorporating Random walk, Random waypoint and Gauss Markov mobility models. The nodes are varied from 10 to 50 . For used three mobility models handoff probability increases with increasing number of nodes. It is concluded that handoff probability is maximum with Gauss Markov mobility model.

\section{Energy Consumption}

Mobile ad hoc networks is a kind of wireless networks which does not depend on the fixed establishments and one node can communicate with other nodes through wireless mode when they are moving. Most of power of nodes in ad hoc networks is supplied with batteries. So, power-saving is of seriousness. Energy consumption of node in network is dependent upon amount of transmission power of node utilized for the transmission of packet. For an ad hoc network each source node transmits data packet to neighboring nodes and these terminals again transmits data packet until the destination node receives it from the corresponding source node. A battery-powered terminal has a limited energy source. Examples of terminals (nodes) in this category are notebook computers, PDAs, and remote controllers. These terminals have a relatively high mobility .For battery-powered terminals the energy for data transmission is limited and power saving is an important feature.

\section{Energy calculations}

The energy consumption is calculated using the Two Ray Ground propagation model for wireless communications. Let $E_{\text {Electric }}$ be the energy dissipated by the transmitter-receiver and $E_{\text {amplifier }}$ be the energy dissipated by the transmit amplifier. Then to transmit $k$ bit message (a packet) a distance $d$ with multi path model, the energy dissipation [6] is

$$
\begin{aligned}
& E_{\text {Transmit }}(k, d)=E_{\text {Electric }}+E_{\text {amplifier }} \times k \times d^{4} \ldots \text { (8) } \\
& E_{\text {receive }}(k)=E_{\text {Electric }} \times k \ldots \ldots \ldots \ldots \ldots \ldots \ldots \ldots \text { (9) }
\end{aligned}
$$

Where $E_{\text {Electric }}$ and $E_{\text {amplifier }}$ have values 50 $n J /$ bit and $0.0013 \mathrm{pJ} / \mathrm{bit} / \mathrm{m}^{4}$ respectively, $k$ is the data rate in bits per packet and $d$ is the distance. There are $n_{i}(i=1,2,3 \ldots \ldots . . . m)$ nodes in the simulator which are initialized with energy levels (Initial_Energy) , Joule and energy saving per node is represented as (Energy_Savings) Joule .After each packet transmission, depending upon the size of the packet in bits and the distance that the packet is sent over, the energy consumed by communication of the packet is respectively deducted from the energy 
of the respective nodes involved in the communication.

The average energy consumed at each node is $\left(\right.$ Average_Energy_consumed $_{i}=E_{\text {Transmit }_{i}}+E_{\text {receive }_{i}}$

The energy savings per node per transmission is calculated as

$\left(\right.$ Energy_Savings $_{i}(k)=\left(\left(\text { Initial_Energy }_{i}\right)_{-}\right.$

$\left.\left(E_{\text {Transmit }}+E_{\text {receive }}\right)_{i(k)}\right)$

In order to achieve quicker communication among the nodes in the entire region, we have partitioned the total service area into four small regions; along with specifically defined handoff region. We have also ensured that only through this handoff region every packet is handed off to another mobile node. This causes reduction in required transmission range and reduction in the value of ' $d$ '. According to the handoff algorithm which has been developed here data is transmitted to the nearest node.

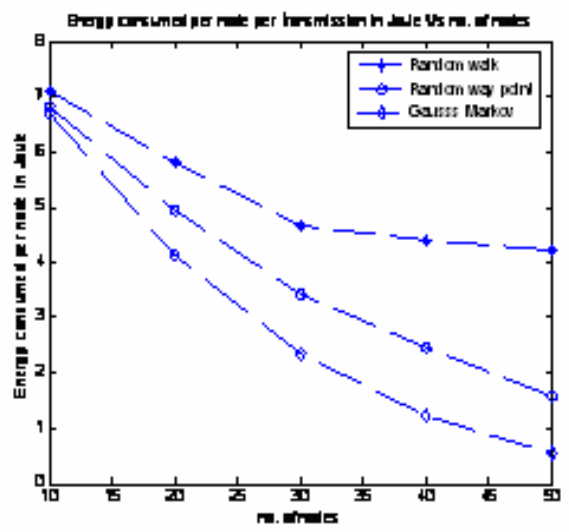

Fig 4- Energy consumed per node per transmission in Joule verses number of nodes

Figure 4 shows Energy consumed per node per transmission in Joule verses number of nodes. As number of nodes in the network increases, value of ' $d$ ' decreases and energy consumed for data transmission and average energy consumed at each node decreases. This in turn increases energy savings at each node. Figure 5 shows energy savings per node per transmission in Joule verses number of nodes. It has been observed that as number of nodes increases energy savings per node per transmission increases. With the Gauss Markov mobility model energy savings per node per transmission is greater as compared to Random walk and Random waypoint mobility models.

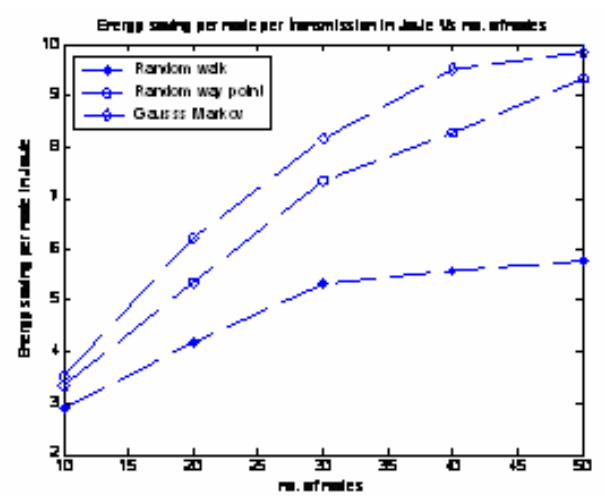

Fig. 5- Energy savings per node per transmission in Joule verses number of nodes

\section{Conclusion}

The handoff algorithm based on Received Signal Strength is used to observe the performance of infrastructure less ad hoc network. From results it can be concluded that handoff probability is greater with Gauss Markov mobility model and less with Random walk mobility model. With Gauss Markov mobility model energy consumed per node per transmission is very less. With Gauss Markov mobility model energy savings per node per transmission is greater. It can be concluded that as node density increases more number of nodes and relays (nodes in handoff region) takes part in the packet transmission process. Hence energy consumed by each node for packet transmission decreases and energy savings at each node increases.

\section{References}

[1] Tracy Camp, Jeff Boleng , Vanessa Davies (2002) Special issue on mobile Ad Hoc Networking: Research, Trends and Applications, 2 (5), 483-502.

[2] Gregory P.Pollini (1996) Trends in Handover design, IEEE Communication Magazine.

[3] Mikael Gudmundson (1991) Analysis of Handover Algorithms, IEEE

[4] Dmitri D. Perkins, Herman D. Hughes, and Charles B. Owen (2002) Factors Affecting the Performance of Ad Hoc Networks, 2048-2052.

[5] Ian F. Akyildiz, Janise McNair, Joseph Ho, Huseyin Uzuinalioglu, and Wenye Wang. Mobility Management in Current and Future Communications Networks.

[6] Muneeb Ali and Zartash Afzal Uzmi. Medium access control with mobilityAdaptive mechanisms for wireless sensor networks. International Journal of. Sensor Networks, 1, 134-142. 